2007s-03

\title{
Iterated Strict Dominance in General Games
}

\author{
Yi-Chun Chen, Ngo Van Long, \\ Xiao Luo
}

\begin{tabular}{c}
\hline Série Scientifique \\
Scientific Series
\end{tabular}

Montréal

Février 2007

(C) 2007 Yi-Chun Chen, Ngo Van Long, Xiao Luo. Tous droits réservés. All rights reserved. Reproduction partielle permise avec citation du document source, incluant la notice (C).

Short sections may be quoted without explicit permission, if full credit, including (C) notice, is given to the source.
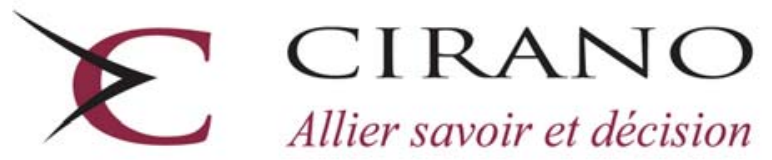

Allier savoir et décision

Centre interuniversitaire de recherche en analyse des organisations 


\section{CIRANO}

Le CIRANO est un organisme sans but lucratif constitué en vertu de la Loi des compagnies du Québec. Le financement de son infrastructure et de ses activités de recherche provient des cotisations de ses organisations-membres, d'une subvention d'infrastructure du Ministère du Développement économique et régional et de la Recherche, de même que des subventions et mandats obtenus par ses équipes de recherche.

CIRANO is a private non-profit organization incorporated under the Québec Companies Act. Its infrastructure and research activities are funded through fees paid by member organizations, an infrastructure grant from the Ministère du Développement économique et régional et de la Recherche, and grants and research mandates obtained by its research teams.

\section{Les partenaires du CIRANO}

Partenaire majeur

Ministère du Développement économique, de l'Innovation et de l'Exportation

\section{Partenaires corporatifs}

\section{Alcan inc.}

Banque de développement du Canada

Banque du Canada

Banque Laurentienne du Canada

Banque Nationale du Canada

Banque Royale du Canada

Banque Scotia

Bell Canada

BMO Groupe financier

Bourse de Montréal

Caisse de dépôt et placement du Québec

DMR Conseil

Fédération des caisses Desjardins du Québec

Gaz de France

Gaz Métro

Hydro-Québec

Industrie Canada

Investissements PSP

Ministère des Finances du Québec

Raymond Chabot Grant Thornton

State Street Global Advisors

Transat A.T.

Ville de Montréal

\section{Partenaires universitaires}

École Polytechnique de Montréal

HEC Montréal

McGill University

Université Concordia

Université de Montréal

Université de Sherbrooke

Université du Québec

Université du Québec à Montréal

Université Laval

Le CIRANO collabore avec de nombreux centres et chaires de recherche universitaires dont on peut consulter la liste sur son site web.

Les cahiers de la série scientifique (CS) visent à rendre accessibles des résultats de recherche effectuée au CIRANO afin de susciter échanges et commentaires. Ces cahiers sont écrits dans le style des publications scientifiques. Les idées et les opinions émises sont sous l'unique responsabilité des auteurs et ne représentent pas nécessairement les positions du CIRANO ou de ses partenaires.

This paper presents research carried out at CIRANO and aims at encouraging discussion and comment. The observations and viewpoints expressed are the sole responsibility of the authors. They do not necessarily represent positions of CIRANO or its partners. 


\title{
Iterated Strict Dominance in General Games
}

\author{
Yi-Chun Chen ${ }^{*}$, Ngo Van Long $^{\dagger}$, Xiao Luo
}

\begin{abstract}
Résumé / Abstract
Nous donnons une définition de l'élimination itérative des stratégies qui sont strictement donimées (EISSD) pour les jeux avec un nombre fini (ou infini) de joueurs, des ensembles de stratégies compactes (ou non-compactes), et des fonctions de gains continues (ou noncontinues). Le processus EISSD est bien défini et indépendant de l'ordre d'élimination. Nous donnons une caractérisation du processus EISSD en utilisant un critère de stabilité et offrons une condition épistémologique. Nous démontrons que le processus EISSD peut produire des équilibres faux dans la classe des jeux de meilleures réponses sécuritaires de Reny. Nous donnons des conditions nécessaires et suffisantes pour que le processus EISSD conserve l’ensemble des équilibre de Nash .
\end{abstract}

Mots clés : théorie des jeux, dominance stricte, élimination itérative, équilibre de Nash, jeux de meilleures réponses sécuritaires de Reny

We offer a definition of iterated elimination of strictly dominated strategies (IESDS) for games with (in)finite players, (non)compact strategy sets, and (dis)continuous payoff functions. IESDS is always a well-defined order independent procedure that can be used to solve Nash equilibrium in dominance-solvable games. We characterize IESDS by means of a "stability" criterion, and offer a sufficient and necessary epistemic condition for IESDS. We show by an example that IESDS may generate spurious Nash equilibria in the class of Reny's better-reply secure games. We provide sufficient/necessary conditions under which IESDS preserves the set of Nash equilibria.

Keywords: game theory, strict dominance, iterated elimination, Nash equilibrium, Reny's better-reply secure games.

Codes JEL : C70, C72

\footnotetext{
* Department of Economics, Northwestern University, Evanston, IL 60208. Email: johnchen@northwestern.edu.

${ }^{\dagger}$ CIRANO and CIREQ, Department of Economics, McGill University, 855 Sherbrooke St West, Montreal, H3A 2T7, Canada. Email: ngo.long@mcgill.ca.

¥ Institute of Economics, Academia Sinica, Taipei 115, Taiwan, ROC. Email: xluo@econ.sinica.edu.tw
} 


\section{Introduction}

Iterated strict dominance is perhaps one of the most basic principles in game theory. The concept of iterated strict dominance rests on the following simple idea: no player would play strategies for which some alternative strategy can yield him/her a greater payoff regardless of what the other players play and this fact is common knowledge. This concept has been used to expound the fundamental conflict between individual and collective rationality as illustrated by the Prisoner's Dilemma, and is closely related to the global stability of the Cournot-tatonnement process in terms of dominance solvability of games (cf. Moulin 1984; Milgrom and Roberts 1990). In particular, it has fruitful applications in Carlsson and van Damme's (1993) global games (see Morris and Shin 2003 for a survey). One paramount advantage of using iterated strict dominance is its extreme simplicity in applications, because it does not entail a probabilistic apparatus.

A variety of elimination procedures has been studied by game theorists. ${ }^{1}$ Among the most interesting questions that have been explored are: Does the order of elimination matter? Is it possible that the iterated elimination process fails to converge to a maximal reduction of a game? What are the sufficient conditions for existence and uniqueness of maximal reduction? Can a maximal reduction generate spurious Nash equilibria?

In the most general setting (where the number of players can be infinite, strategy sets can be in general topological spaces, and payoff functions can be discontinuous) Dufwenberg and Stegeman (2002) (henceforth DS) investigated the properties of a definition of iterated elimination of (strictly) dominated strategies (IESDS). Among others, DS demonstrated that (i) IESDS

\footnotetext{
${ }^{1}$ See in particular Moulin (1984), Gilboa, Kalai, and Zemel (1990), Stegeman (1990), Milgrom and Roberts (1990), Borgers (1993), Lipman (1994), Osborne and Rubinstein (1994), among others. (See also Jackson (1992) and Marx and Swinkels (1997) for iterated weak dominance.)
} 
is in general an order dependent procedure, (ii) a maximal reduction may fail to exist, and (iii) IESDS can generate spurious Nash equilibria even in "dominance-solvable" games. ${ }^{2}$ As DS pointed out, these anomalies and pathologies appear to be rather surprising and somewhat counterintuitive. In particular, outside the class of games with finite players, compact strategy sets, and continuous payoff functions, DS's IESDS procedure is not well defined because of the lack of the existence of a maximal reduction. DS (2002, p. 2022) concluded that:

The proper definition and role of iterated strict dominance is unclear for games that are not compact and continuous. ... The identification of general classes of games for which IESDS is an attractive procedure, outside of the compact and continuous class, remains an open problem.

The main purpose of this paper is to offer a new definition of IESDS that is suitable for all games, possibly with an arbitrary number of players, arbitrary strategy sets, and arbitrary payoff functions. This definition of IESDS will be denoted by IESDS* (the asterisk $*$ is used to distinguish it from other forms of IESDS). We will show that IESDS* is a well-defined order independent procedure: it yields a unique maximal reduction (see Theorem 1). This nice property is completely topology-free. For games that are compact and continuous, our IESDS* yields the same maximal reduction as DS's definition of IESDS (see Theorem 2). We provide a characterization of IESDS* in terms of a "stability" criterion (see Theorem 3). We also provide within the semantic framework of knowledge, a sufficient and necessary epistemic condition for the concept of IESDS* (see Theorem 4).

The IESDS* proposed in this paper is based mainly upon Milgrom and Roberts's (1990, pp. 1264-1265) definition of IESDS in a general class of

\footnotetext{
${ }^{2}$ DS also provided sufficient conditions for positive results. In particular, if strategy spaces are compact Hausdorff spaces and payoff functions are continuous, then DS's definition of IESDS yields a unique maximal reduction.
} 
supermodular games, and has two major features: (1) IESDS* allows for an uncountable number of rounds of elimination, and is thus more general than DS's IESDS procedure, and (2) in each round of elimination, IESDS* allows for eliminating dominated strategies (possibly by using strategies that have previously been eliminated), rather than eliminating only those strategies that are dominated by some uneliminated strategy. Thus, the effect of successive rounds of elimination is only to narrow the set of beliefs about one's opponents, not to narrow the range of choices available to the player. Consequently, these two features endow the IESDS* procedure with greater elimination power than DS's IESDS procedure.

The rationale behind the two features of IESDS* is as follows. Recall that a prominent justification for IESDS is "common knowledge of rationality"; see, e.g., Bernheim (1984), Osborne and Rubinstein (1994, Chapter 4), Pearce (1984), and Tan and Werlang (1988). While the equivalence between IESDS and the strategic implication of "common knowledge of rationality" has been established for games with compact strategy spaces and continuous payoff functions (see Bernheim 1984, Proposition 3.1), Lipman (1994) demonstrated that, for a more general class of games, there is a non-equivalence between countably infinite iterated elimination of never-best replies and the strategic implication of "common knowledge of rationality". Lipman (1994, Theorem 2) showed that the equivalence can be restored by "removing never best replies as often as necessary" (p. 122), i.e., by allowing for an uncountably infinite iterated elimination of never-best replies. ${ }^{3}$ Therefore, it seems fairly natural and desirable to define IESDS for general games by allowing for an uncountably infinite iterated elimination. Example 1 in Section 2 shows that IESDS* is necessarily defined by an uncountably infinite number of

\footnotetext{
${ }^{3}$ However, in general games with noncompact strategy sets or discontinuous payoff functions, a never-best strategy might fail to be a strictly dominated strategy; see Bergemann and Morris (2005, Footnote 8) for such an example. We thank Stephen Morris for drawing our attention to this point.
} 
elimination rounds.

The second feature of IESDS* is in the same spirit as Milgrom and Roberts's (1990, pp. 1264-1265) definition of IESDS. ${ }^{4}$ That is, in each round of elimination, IESDS* allows for eliminating dominated strategies, rather than eliminating only those strategies that are dominated by some uneliminated strategy in that round. For games where strategy spaces are compact and payoff functions are uppersemicontinuous in own strategies, this feature does not imply giving IESDS* more elimination power than DS's IESDS procedure, because it can be shown that, in this class of games, for any dominated strategy, there is some remaining uneliminated strategy that dominates it (see DS's Lemma, p. 2012). ${ }^{5}$ However, for more general games, the second feature of IESDS* gives it more elimination power than DS's IESDS procedure; examples can be easily found to show that our IESDS* procedure converges much faster than DS's IESDS procedure. ${ }^{6}$

To illustrate our basic points, consider a simple one-person game where the strategy space is $(0,1)$ and the payoff function is $u(x)=x$ for every strategy $x$. (This game is also described in DS's Example 5, p. 2011.) Clearly, every strategy is a never-best reply and is dominated only by a dominated

\footnotetext{
${ }^{4}$ Formally, given any product subset $\widehat{S}$ of strategy profiles, Milgrom and Roberts (1990, p. 1265) defined the set of player $i$ 's undominated responses to $\widehat{S}$ as including strategies of $i$ that are undominated by not only uneliminated strategies, but also by previously eliminated strategies. From the viewpoint of learning theory, the second feature of IESDS* can be "justified" by Milgrom and Roberts's (1990, p. 1269) adaptive learning process, where each player will never play a strategy for which there is another strategy, from the player's strategy space, that would have done better against every combination of the other players' strategies in the recent past plays. Ritzberger (2002, Section 5.1) also considered a similar definition of IESDS for compact and continuous games that allows for eliminating strategies that are dominated by an uneliminated or eliminated strategy. We are grateful to Martin Dufwenberg for drawing our attention to this.

${ }^{5}$ Milgrom and Roberts (1996, Lemma 1, p. 117) showed an analogous result which allows for dominance by mixed strategy. Chen and Luo (2003, Lemma 5) proved a similar result by using Zorn's Lemma.

${ }^{6}$ The study of computable algorithms to determine the limit outcome of IESDS* is certainly an interesting problem worthy of further investigation, but beyond the scope of this paper.
} 
strategy. Eliminate in round one all strategies except a particular strategy $x$ in $(0,1)$. In particular, under DS's IESDS procedure, $x$ survives DS's IESDS and is thus a "spurious Nash equilibrium" - i.e. a Nash equilibrium of the reduced game after iterated elimination of strictly dominated strategies, which is not a Nash equilibrium of the original game. Under our IESDS $^{*}$, in round two, $x$ is further eliminated, and thus our maximal reduction yields an empty set of strategies, indicating (correctly) that the game has no Nash equilibrium. This makes sense since $x$ cannot be justified as a best reply (and hence cannot be justified by any higher order knowledge of "rationality"). Consequently, this example shows that eliminating dominated strategies, rather than eliminating only those strategies that are dominated by some uneliminated strategy or by some undominated strategy, is a very natural and desirable requirement for a definition of IESDS in general games; see also our Example 2 in Section $2 .^{7}$

We also study the relationship between Nash equilibria and IESDS* Example 4 in Section 4 demonstrates that, even with its strong elimination power, our IESDS* can generate spurious Nash equilibria. In particular, the game in Example 4 is in the class of Reny's (1999) better-reply secure games, which have regular properties such as compact and convex strategy spaces, as well as quasi-concave and bounded payoff functions. We offer a sufficient

\footnotetext{
${ }^{7}$ The conventional notion of rationality requires that an individual's choice be optimal within the feasible choice set given his information; see Aumann (1987), Aumann and Brandenburger (1995), Bernheim (1984), Brandenburger and Dekel (1987), Epstein (1997), and Tan and Werlang (1988). In the case of finite games, it is easy to see that $n$-level justifiable* strategy (meaning a player's choice is optimal in the player's feasible

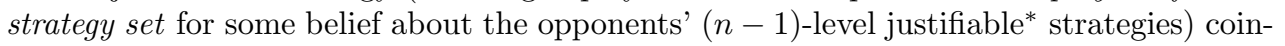
cides with $n$-level justifiable strategy (meaning a player's choice is optimal in the player's $(n-1)$-level justifiable strategy set for some belief about the opponents' $(n-1)$-level justifiable strategies); see Pearce (1984, Proposition 2) and Osborne and Rubinstein (1994, Proposition 61.2). This coincidence makes it possible to define an alternative iteration for finite games by gradually reduced subgames. However, Osborne and Rubinstein (1994, Definitions 54.1 and 55.1) define rationalizability by the standard "best responses" over the set of all feasible strategies.
} 
and necessary condition of no spurious Nash equilibria. In particular, no spurious Nash equilibria appear in one-person or "dominance solvable" games (see Theorem 5). In addition, no spurious Nash equilibria appear in many games that arise in economic applications (see Corollary 4).

The remainder of this paper is organized as follows. Section 2 offers the definition of IESDS* and investigates its properties. Section 3 provides epistemic foundations for IESDS*. Section 4 studies the relationship between IESDS* and Nash equilibria. Section 5 offers some concluding remarks.

\section{IESDS*}

Throughout this paper, we consider a strategic game $\mathcal{G} \equiv\left(N,\left\{X_{i}\right\}_{i \in N},\left\{u_{i}\right\}_{i \in N}\right)$, where $N$ is an arbitrary set of players, for each $i \in N, X_{i}$ is an arbitrary set of player $i$ 's strategies, and $u_{i}: X_{i} \times X_{-i} \rightarrow \Re$ is $i$ 's arbitrary payoff function. $X \equiv \prod_{i \in N} X_{i}$ is the joint strategy set. A strategy profile $x^{*} \in X$ is said to be a Nash equilibrium if for every $i, x_{i}^{*}$ maximizes $u_{i}\left(., x_{-i}^{*}\right)$.

A strategy $x_{i} \in X_{i}$ is said to be dominated given $Y \subseteq X$ if for some strategy $x_{i}^{\prime} \in X_{i}{ }^{8} u_{i}\left(x_{i}^{\prime}, y_{-i}\right)>u_{i}\left(x_{i}, y_{-i}\right)$ for all $y_{-i} \in Y_{-i}$, where $Y_{-i} \equiv\left\{y_{-i} \mid\right.$ $\left.\left(y_{i}, y_{-i}\right) \in Y\right\}$.

The following example illustrates that for some games, our IESDS* (a formal definition of which will be given below) yields a maximal reduction containing all Nash equilibria (in this case, a singleton) only after an uncountably infinite number of rounds. This is unlike Lipman's (1994) Example and

\footnotetext{
${ }^{8}$ In the literature, especially in the case of finite games, a dominated (pure) strategy is normally defined by the existence of a mixed strategy that generates a higher expected payoff against any strategy profile of the opponents. In this paper, we follow DS in defining, rather conservatively, a dominated (pure) strategy by the existence of a (pure) strategy that generates a higher payoff against any strategy profile of the opponents. The two definitions of dominance are equivalent for games where strategy spaces are convex; for instance, mixed extensions of finite games. Borgers (1993) provided an interesting justification for "pure strategy dominance" by viewing players' payoff functions as preference orderings over the pure strategy outcomes of the game.
} 
DS's Examples 3 and 6, which can be remedied to yield a maximal reduction by performing a second countable elimination after a first countable elimination. To the best of our knowledge, this example is the first one to show that a sensible maximal reduction should not only go beyond a countable elimination, but also must go to an uncountable elimination.

Example 1. Consider a two-person symmetric game: $\mathcal{G} \equiv\left(N,\left\{X_{i}\right\}_{i \in N},\left\{u_{i}\right\}_{i \in N}\right)$, where $N=\{1,2\}, X_{1}=X_{2}=[0,1]$, and for all $x_{i}, x_{j} \in[0,1], i, j=1,2$, and $i \neq j$

$$
u_{i}\left(x_{i}, x_{j}\right)=\left\{\begin{array}{ll}
1, & \text { if } x_{i}=1 \\
2, & \text { if } x_{i} \succ x_{j} \text { and } x_{i} \neq 1 \\
0, & \text { if } x_{i} \prec x_{j} \text { or } x_{i}=x_{j} \neq 1
\end{array},\right.
$$

where $\preccurlyeq$ is a linear order on $[0,1]$ satisfying (i) 1 is the greatest element; and (ii) $[0,1]$ is well ordered by the linear order $\preccurlyeq .^{9}$

In this example only the least element $r^{0}$ in $[0,1]$ (w.r.t. $\left.\preccurlyeq\right)$ is strictly dominated by 1 . After eliminating $r^{0}$ from [0,1], only the least element $r^{1}$ in $[0,1] \backslash\left\{r^{0}\right\}$ is strictly dominated by 1 given $[0,1] \backslash\left\{r^{0}\right\}$. It is easy to see that every strategy is eliminated whenever every smaller strategy is eliminated and only one element in $[0,1]$ is eliminated at each round. Thus, IESDS* leads to a unique uncountable elimination, which leaves only the greatest element 1 for each player. ${ }^{10}$

\footnotetext{
${ }^{9} \mathrm{~A}$ linear order is a complete, reflexive, transitive, and antisymmetric binary relation. A set is said to be well ordered by a linear order if each of its nonempty subsets has a least or first element. By the well-ordering principle - i.e., every nonempty set can be well ordered (see, e.g., Aliprantis and Border 1999, Section 1.12), [0,1] can be well ordered by a linear order $\preccurlyeq$ with the great element of 1 . Note that the linear order $\preccurlyeq$ used in our Example 1 is not the "natural" order on $[0,1]$. (One can construct such an example by using the fact that there exists an uncountable well-ordered set without referring to the choice axiom or the well-ordering principle. We thank Kim-Sau Chung for pointing this out to us.)

${ }^{10}$ Example 1 also illustrates that DS's IESDS procedure may fail to yield a maximal reduction. DS's Theorem 1 on existence and uniqueness of maximal reduction relies on the game $\mathcal{G}$ being a compact and continuous game, which is not the case in our example (because it is impossible to find a topology on $[0,1]$ such that $\mathcal{G}$ is a compact and continuous game).
} 
Let us proceed to a formal definition of our IESDS*. For any subsets $Y, Y^{\prime} \subseteq X$ where $Y^{\prime} \subseteq Y$, we use the notation $Y \rightarrow Y^{\prime}$ (read: $Y$ is reduced to $Y^{\prime}$ ) to signify that for any $y \in Y \backslash Y^{\prime}$, some $y_{i}$ is dominated given $Y$. Let $\lambda^{0}$ denote the first element in an ordinal $\Lambda$, and let $\lambda+1$ denote the successor to $\lambda$ in $\Lambda .^{11}$

Definition. An iterated elimination of strictly dominated strategies (IESDS*) is defined as a finite, countably infinite, or uncountably infinite family $\left\{\mathcal{D}^{\lambda}\right\}_{\lambda \in \Lambda}$ such that $\mathcal{D}^{\lambda^{0}}=X, \mathcal{D}^{\lambda} \rightarrow \mathcal{D}^{\lambda+1}$ (and $\mathcal{D}^{\lambda}=\cap_{\lambda^{\prime}<\lambda} \mathcal{D}^{\lambda^{\prime}}$ for a limit ordinal $\lambda$ ), and $\mathcal{D} \equiv \cap_{\lambda \in \Lambda} \mathcal{D}^{\lambda} \rightarrow \mathcal{D}^{\prime}$ only for $\mathcal{D}^{\prime}=\mathcal{D}$. The set $\mathcal{D}$ is called a "maximal reduction."

The above definition of IESDS* does not require the elimination of all dominated strategies in each round of elimination. That is, we do not require that for every $\lambda, \mathcal{D}^{\lambda+1}=\mathcal{D}^{\lambda} \backslash\left\{y \in \mathcal{D}^{\lambda} \mid \exists i\right.$ s.t. $y_{i}$ is dominated given $\left.\mathcal{D}^{\lambda}\right\}$. This flexibility raises an important question: does the IESDS* procedure yield a unique maximal reduction? Without imposing any topological condition on the games, we show that IESDS* is always a well-defined order independent procedure and $\mathcal{D}$ is nonempty if a Nash equilibrium exists. Formally, we have:

Theorem $1 \mathcal{D}$ exists and is unique. Moreover, $\mathcal{D}$ is nonempty if the game $\mathcal{G}$ has a Nash equilibrium.

To prove Theorem 1, we need the following two lemmas. These lemmas are mainly consequences of the feature of IESDS* that the procedure allows for eliminating dominated strategies possibly by using strategies that have previously been eliminated.

\footnotetext{
${ }^{11}$ An ordinal $\Lambda$ is a well-ordered set in the order-isomorphic sense (see, e.g., Suppes 1972 , p. 131). In particular, the well-ordered set of natural numbers is called the first infinite ordinal. A limit ordinal is an element in $\Lambda$ which is not a successor. As usual, we use $\lambda^{\prime}<\lambda$ to mean that " $\lambda^{\prime}$ precedes $\lambda$."
} 
Lemma 1 For every $x \in \mathcal{D}$ and every $i, x_{i}$ is not dominated given $\mathcal{D}$.

Proof. Assume, in negation, that for some $y \in \mathcal{D}$ and some $i, y_{i}$ is dominated given $\mathcal{D}$. Thus, $\mathcal{D} \rightarrow \mathcal{D} \backslash\{y\} \neq \mathcal{D}$, which is a contradiction.

Lemma 2 For any $Y \subseteq Y^{\prime}$, a strategy is dominated given $Y$ if it is dominated given $Y^{\prime}$.

Proof. Let $y_{i}$ be a strategy that is dominated given $Y^{\prime}$. That is, $u_{i}\left(x_{i}, y_{-i}\right)>$ $u_{i}\left(y_{i}, y_{-i}\right)$ for some $x_{i} \in X_{i}$ and all $y_{-i} \in Y_{-i}^{\prime}$. Since $Y \subseteq Y^{\prime}, u_{i}\left(x_{i}, y_{-i}\right)>$ $u_{i}\left(y_{i}, y_{-i}\right)$ for all $y_{-i} \in Y_{-i}$. Therefore, $y_{i}$ is dominated given $Y$.

We now turn to the proof of Theorem 1.

Proof of Theorem 1. For any $Y \subseteq X$ we define the "next elimination" operation $\nabla$ by

$$
\nabla[Y] \equiv\left\{y \in Y \mid \exists i \text { s.t. } y_{i} \text { is dominated given } Y\right\}
$$

By the well-ordering principle, the power set of $X$ can be well ordered by a linear order; cf. e.g. Aliprantis and Border (1999, Chapter 1). The existence of a maximal reduction using IESDS* is assured by the following prominent "fast" IESDS*: $\mathcal{D} \equiv \cap_{\lambda \in \Lambda} \mathcal{D}^{\lambda}$ satisfying $\mathcal{D}^{\lambda^{0}}=X, \mathcal{D}^{\lambda+1}=\mathcal{D}^{\lambda} \backslash \nabla\left[\mathcal{D}^{\lambda}\right]$, and $\mathcal{D}^{\lambda}=\cap_{\lambda^{\prime}<\lambda} \mathcal{D}^{\lambda^{\prime}}$ for a limit ordinal $\lambda$, where $\Lambda$ is an ordinal that is order-isomorphic to the power set of $X$. Note that $\nabla\left[\mathcal{D}^{\lambda}\right]=\varnothing$ implies $\nabla\left[\mathcal{D}^{\lambda^{\prime}}\right]=\varnothing$ for all $\lambda^{\prime}>\lambda$. By using the fact that a set is never isomorphic to its power set (cf. e.g. Suppes 1972, Theorem 23), it is easily verified that $\mathcal{D} \rightarrow \mathcal{D}^{\prime}$ only for $\mathcal{D}=\mathcal{D}^{\prime}$.

Now suppose that $\mathcal{D}$ and $\mathcal{D}^{\prime}$ are two maximal reductions obtained by applying IESDS* procedure. Since $\mathcal{D} \cup \mathcal{D}^{\prime} \subseteq \mathcal{D}^{\lambda^{0}}$, by Lemmas 1 and 2, $\mathcal{D} \cup \mathcal{D}^{\prime} \subseteq \mathcal{D}^{\lambda}$ for all $\lambda$. Therefore, $\mathcal{D} \cup \mathcal{D}^{\prime} \subseteq \mathcal{D}$. Similarly, $\mathcal{D} \cup \mathcal{D}^{\prime} \subseteq \mathcal{D}^{\prime}$. Thus, $\mathcal{D}=\mathcal{D}^{\prime}$. Let $x^{*}$ be a Nash equilibrium. Since for every $i, x_{i}^{*}$ is not dominated given $\left\{x^{*}\right\}$, by Lemma $2, x^{*} \in \mathcal{D}^{\lambda}$ for all $\lambda$. 
An immediate corollary of the proof of Theorem 1 is as follows:

Corollary 1. Every Nash equilibrium survives both IESDS* procedure and $D S$ 's IESDS procedure (if exists).

Proof. Let $\mathcal{H}$ be the maximal reduction resulting from an IESDS procedure in the DS sense. Since every strategy that is dominated by an uneliminated strategy is a dominated strategy, by Theorem 1 , the unique $\mathcal{D} \subseteq \mathcal{H}$. By the proof of Theorem 1, every Nash equilibrium survives $\mathcal{D}$ and hence, survives $\mathcal{H}$.

In contrast to DS's IESDS, our IESDS* does not require that, in each round of elimination, the dominator of an eliminated strategy be some uneliminated strategy. However, the following result asserts that, for the class of games where strategy spaces are compact (Hausdorff) and payoff functions are own-uppersemicontinuous (i.e. uppersemicontinuous in own strategies), any maximal reduction of $\mathcal{G}$ using DS's IESDS procedure yields a joint strategy set identical to our $\mathcal{D}$. Thus, our IESDS* extends DS's IESDS to arbitrary games. Let $\mathcal{H}$ denote a maximal reduction of $\mathcal{G}$ in the DS sense, i.e., a set of strategy profiles resulting from using DS's IESDS procedure. Formally, we have:

Theorem 2 For any compact and own-uppersemicontinuous game, $\mathcal{H}=\mathcal{D}$ if $\mathcal{H}$ exists. Moreover, for any compact (Hausdorff) and continuous game, $\mathcal{H}=\mathcal{D}$.

Proof. Suppose that $\mathcal{H}$ is the maximal reduction resulting from an IESDS procedure in the DS sense. Clearly, $\mathcal{D}=\emptyset$ if $\mathcal{H}=\emptyset$. By DS's Lemma, for any $i$ and any $x \in \mathcal{H} \neq \emptyset, x_{i}$ is not dominated given $\mathcal{H}$. According to Definition in this paper, $\mathcal{H}=\mathcal{D}$. Moreover, by DS's Theorem $1(\mathrm{~b}), \mathcal{H}$ exists if the game is compact and continuous. The last part of Theorem 2 follows immediately from the first part and from DS's Theorem 1. 
The following example demonstrates that outside the class of compact and own-uppersemicontinuous games, $\mathcal{D}$ could be very different from a unique $\mathcal{H}$ that results from a well-defined "fast" IESDS procedure in the DS sense.

Example 2. Consider a two-person symmetric game: $\mathcal{G}=\left(N,\left\{X_{i}\right\}_{i \in N},\left\{u_{i}\right\}_{i \in N}\right)$, where $N=\{1,2\}, X_{1}=X_{2}=[0,1]$, and for all $x_{i}, x_{j} \in[0,1], i, j=1,2$, and $i \neq j$ (cf. Fig. 1)

$$
u_{i}\left(x_{i}, x_{j}\right)= \begin{cases}x_{i}, & \text { if } x_{i}<\frac{1}{2} \text { or } x_{j}=\frac{1}{2} \\ \frac{1}{2} \min \left\{x_{i}, x_{j}\right\}, & \text { if } x_{i} \geq \frac{1}{2} \text { and } x_{j}>\frac{1}{2} \\ 0, & \text { if } x_{i} \geq \frac{1}{2} \text { and } x_{j}<\frac{1}{2}\end{cases}
$$

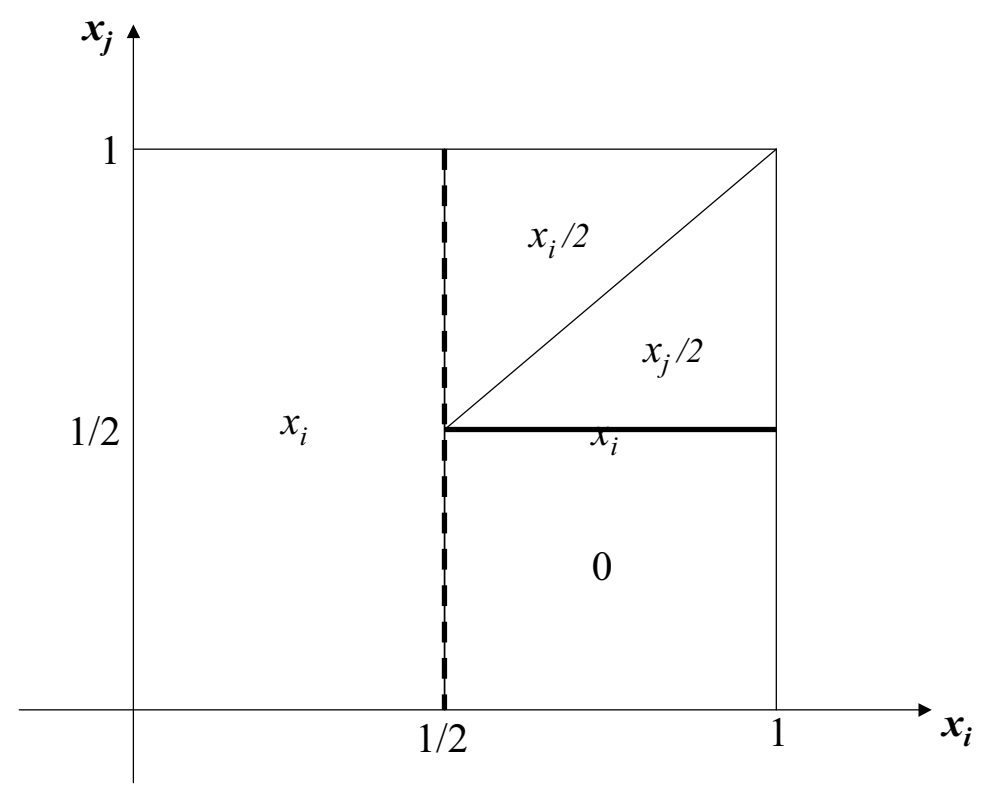

Fig. 1. Payoff function $u_{i}\left(x_{i}, x_{j}\right)$.

In this game it is easy to see that any strategy $x_{i}$ in $[0,1 / 2)$ is dominated by $y_{i}=\frac{1}{4}+\frac{x_{i}}{2}>x_{i}$ for $x_{i}<\frac{1}{2}$. After eliminating all these dominated strategies, $1 / 2$ is dominated by 1 since (i) $u_{i}(1,1 / 2)=1>1 / 2=u_{i}(1 / 2,1 / 2)$ 
if $x_{j}=1 / 2$, and (ii) $u_{i}\left(1, x_{j}\right)=x_{j} / 2>1 / 4=u_{i}\left(1 / 2, x_{j}\right)$ if $x_{j}>1 / 2$. After eliminating the strategy $1 / 2$, no $x_{i} \in(1 / 2,1]$ is strictly dominated by some strategy $x_{i}^{\prime} \in(1 / 2,1]$, because in the joint strategy set $(1 / 2,1] \times(1 / 2,1]$, setting $x_{j}=x_{i}$, we have $u_{i}\left(x_{i}, x_{j}\right)=x_{i} / 2 \geq u_{i}\left(x_{i}^{\prime}, x_{j}\right)$ for all $x_{i}^{\prime} \in(1 / 2,1]$. Thus, $\mathcal{H} \equiv(1 / 2,1] \times(1 / 2,1]$ is the unique maximal reduction under the "fast" IESDS procedure in the DS sense.

However, any $x_{i} \in(1 / 2,1)$ is dominated by the previously eliminated strategy $y_{i}=\left(1+x_{i}\right) / 4 \in[0,1 / 2)$ since, for all $x_{j} \in(1 / 2,1], u_{i}\left(x_{i}, x_{j}\right) \leq$ $x_{i} / 2<\left(1+x_{i}\right) / 4=u_{i}\left(y_{i}, x_{j}\right)$. Thus, $\mathcal{D}=\{(1,1)\} \neq \mathcal{H}$. In fact, $(1,1)$ is the unique Nash Equilibrium, which could also be obtained with the "iterated elimination of never-best replies" (cf., e.g., Bernheim 1984; Lipman 1994). In this game, the payoff function $u_{i}\left(., x_{j}\right)$ is not uppersemicontinuous since $\lim \sup _{x_{i} \uparrow 1 / 2} u_{i}\left(x_{i}, x_{j}\right)=1 / 2>0=u_{i}\left(1 / 2, x_{j}\right)$ for all $x_{j}<1 / 2$.

We next turn to providing a characterization of IESDS* by means of a "stability" criterion. A subset $\mathcal{K} \subseteq X$ is said to be a stable set if $\mathcal{K}=\{x \in X \mid$ $x_{i}$ is not dominated given $\left.\mathcal{K}\right\}$; cf. Luo (2001, Definition 3). Clearly, a stable set must be in Cartesian product form.

Theorem $3 \mathcal{D}$ is the largest stable set.

Proof. By Lemma 1, for all $x \in \mathcal{D}$, each player $i$ 's strategy $x_{i}$ is undominated given $\mathcal{D}$. By Lemma 2, for all $y \in X \backslash \mathcal{D}$, some player $i$ 's strategy $y_{i}$ is dominated given $\mathcal{D}$. Consequently, $\mathcal{D}$ is a stable set. However, by Lemma 2 , every stable set $\mathcal{K} \subseteq \mathcal{D}^{\lambda}$ for all $\lambda$. Hence, $\mathcal{D} \equiv \cap_{\lambda \in \Lambda} \mathcal{D}^{\lambda}$ is the largest stable set.

The following result is an immediate corollary of Theorem 3.

Corollary 2. $D S$ 's IESDS is order independent if every $\mathcal{H}$ is a stable set. Proof. Let $\mathcal{H}$ be a maximal reduction resulting from an IESDS procedure in the DS sense. It suffices to show that $\mathcal{H}=\mathcal{D}$. Since $\mathcal{H}$ is a stable set, 
by Theorem $3, \mathcal{H} \subseteq \mathcal{D}$. By the proof of Corollary $1, \mathcal{D} \subseteq \mathcal{H}$. Therefore, $\mathcal{H}=\mathcal{D}$.

Corollary 2 does not require the game $\mathcal{G}$ to have compact strategy sets or uppersemicontinuous payoff functions. Of course, if strategy spaces are compact (Hausdorff) and payoff functions are uppersemicontinuous in own strategies, then by DS's Lemma, every dominated strategy has an undominated dominator. Under these conditions, every maximal DS's reduction $\mathcal{H}$ is a stable set. Corollary 2 therefore generalizes DS's Theorem 1(a). The following example illustrates this point.

Example 3. Consider a two-person game: $\mathcal{G}=\left(N,\left\{X_{i}\right\}_{i \in N},\left\{u_{i}\right\}_{i \in N}\right)$, where $N=\{1,2\}, X_{1}=X_{2}=[0,1]$, and for all $x_{1}, x_{2} \in[0,1], u_{1}\left(x_{1}, x_{2}\right)=x_{1}$ and

$$
u_{2}\left(x_{1}, x_{2}\right)=\left\{\begin{array}{ll}
x_{2}, & \text { if } x_{2}<1 \\
1, & \text { if } x_{1}=1 \text { and } x_{2}=1 \\
0, & \text { if } x_{1}<1 \text { and } x_{2}=1
\end{array} .\right.
$$

In this example it is easy to see that $\{(1,1)\}$ is the unique maximal reduction under DS's IESDS procedure, and that it is a stable set. Thus, DS's IESDS procedure is order independent in this game. However, $u_{2}\left(x_{1},.\right)$ is not uppersemicontinuous in $x_{2}$ at $x_{2}=1$ if $x_{1} \neq 1$ and hence DS's Theorem 1(a) does not apply.

Gilboa, Kalai, and Zemel (1990) (GKZ) considered a variety of elimination procedures. GKZ's definition of IESDS requires that in each round of elimination, any eliminated strategy is dominated by a strategy which is not eliminated in that round of elimination (see DS 2002, pp. 2018-2019). An immediate corollary of Theorems 1 and 3 is as follows.

Corollary 3. (i) GKZ's IESDS procedure is order independent if every maximal reduction under GKZ's IESDS procedure is a stable set. (ii) For 
any compact and (own-uppersemi) continuous game, GKZ's IESDS procedure (if exists) yields the same $\mathcal{D}$.

Proof. The proof of the first part is totally similar to the proof of Corollary 2. Now suppose that the game is compact and (own-uppersemi)continuous, and suppose that $\mathcal{H}$ is a maximal reduction resulting from an IESDS procedure in the GKZ sense. Clearly, $\mathcal{D}=\emptyset$ if $\mathcal{H}=\emptyset$. By DS's Lemma and DS's Theorem 3, GKZ's definition of IESDS coincides with DS's definition of IESDS. By Theorem $2, \mathcal{H}=\mathcal{D}$.

\section{Epistemic Foundations of IESDS*}

In this section we present an epistemic characterization for IESDS*. Our approach here is in the same spirit as Lipman's (1994) epistemic characterization for the iterated elimination of "never-best" strategies. More specifically, we offer a sufficient and necessary condition for the concept of iterated strict dominance: no player would play strategies for which some alternative strategy can yield him/her a greater payoff regardless of what the other players play and this fact is common knowledge.

Note that every strictly dominated strategy must be a never-best response, although a never-best response might fail to be a strictly dominated strategy (see Footnote 3). That is, Bayesian rationality implies that no player uses a strictly dominated strategy. This implication is also true for other notions of rationality other than subjective expected utility maximization, e.g. probabilistically sophisticated preferences, the multi-priors model, and monotonic preferences; see Epstein (1997) for extensive discussions. Thus, we may take the requirement of using no strictly dominated strategy as a more primitive notion of rationality. Following Aumann (1976, 1987, 1995, and 1999), we establish below, within the standard semantic framework, some epistemic foundation for IESDS* by using this notion of rationality in terms 
of payoff dominance.

Consider a model of knowledge for a game $\mathcal{G}:^{12} \mathcal{M}(\mathcal{G}) \equiv\left(\Omega,\left\{P_{i}\right\}_{i \in N},\left\{\chi_{i}\right\}\right)$, where $\Omega$ is the space of states with typical element $\omega \in \Omega, P_{i}(\omega) \subseteq \Omega$ is player $i$ 's information structure at $\omega$, and $\chi_{i}(\omega) \in X_{i}$ is $i$ 's strategy at $\omega$. Let $\chi(\omega) \equiv\left(\chi_{i}(\omega), \chi_{-i}(\omega)\right)$. For event $E \subseteq \Omega, i$ knows $E$ at $\omega$ if $P_{i}(\omega) \subseteq E$. Let $K_{i} E \equiv\{\omega \in \Omega \mid i$ knows $E$ at $\omega\}$. Say $i$ is rational ${ }^{*}$ at $\omega$ if $\exists y_{i} \in X_{i}$ such that $u_{i}\left(y_{i}, \chi_{-i}\left(\omega^{\prime}\right)\right)>u_{i}\left(\chi_{i}(\omega), \chi_{-i}\left(\omega^{\prime}\right)\right)$ for all $\omega^{\prime} \in P_{i}(\omega)$, i.e., $i$ 's strategy $\chi_{i}(\omega)$ is not strictly dominated given $\left\{\chi\left(\omega^{\prime}\right) \mid \omega^{\prime} \in P_{i}(\omega)\right\}$. Let $R_{i}^{*} \equiv\left\{\omega \in \Omega \mid i\right.$ is rational ${ }^{*}$ at $\left.\omega\right\}$, and $R^{*} \equiv \cap_{i \in N} R_{i}^{*}$. Let $R^{*}$ denote an arbitrary self-evident event in $R^{*}$, i.e., $R^{*} \subseteq\left(R^{*} \cap K_{i} R^{*}\right) \forall i \in N$. It is by now well-known that $\omega \in R^{*}$ iff rationality* is common knowledge at $\omega$. The following Theorem 4 states that IESDS* is the strategic implication of common knowledge of rationality*.

Theorem 4 There is a model of knowledge such that $x \in \mathcal{D}$ iff $x=\chi(\omega)$ for some $\omega \in R^{*}$. Moreover, for any model of knowledge, $\chi(\omega) \in \mathcal{D}$ for all $\omega \in R^{*}$.

Proof. Define $\Omega \equiv \mathcal{D}$. For any $\omega=\left(x_{i}\right)_{i \in N}$ in $\Omega$, define $\chi_{i}(\omega)=x_{i} \forall i$ and $P_{i}(\omega)=\left\{\omega^{\prime} \in \Omega \mid \chi_{i}\left(\omega^{\prime}\right)=\chi_{i}(\omega)\right\} \forall i$. Clearly, $\left\{\chi_{-i}\left(\omega^{\prime}\right) \mid \omega^{\prime} \in P_{i}(\omega)\right\}=$ $\mathcal{D}_{-i}$. Since by Theorem $3, \mathcal{D}$ is a stable set in Cartesian product form, every strategy $\chi_{i}(\omega)$ is not strictly dominated given $\left\{\chi\left(\omega^{\prime}\right) \mid \omega^{\prime} \in P_{i}(\omega)\right\}$, and hence, $i$ is rational* at every $\omega \in \Omega$. Therefore, $\Omega$ is a self-evident event in $R^{*}$, i.e. $\Omega=R^{*}$.

Now, consider a self-evident event $R^{*}$ in any given model of knowledge $\mathcal{M}(\mathcal{G})$. Let $Z \equiv\left\{\chi\left(\omega^{\prime}\right) \mid \omega^{\prime} \in R^{*}\right\}$. By rationality*, we know that for any $\omega^{\prime} \in R^{*}$ the strategy $\chi_{i}\left(\omega^{\prime}\right)$ is not strictly dominated given $\left\{\chi\left(\omega^{\prime}\right) \mid \omega^{\prime} \in P_{i}(\omega)\right\}$. Since $R^{*}$ is self-evident we have $P_{i}(\omega) \subseteq R^{*}$, and thus, by Lemma $2, \chi_{i}\left(\omega^{\prime}\right)$

\footnotetext{
${ }^{12}$ See, e.g., Aumann (1999) and Osborne and Rubinstein (1994, Chapter 5).
} 
is not strictly dominated given $Z$. Again by Lemma $2, Z \subseteq \mathcal{D}^{\lambda}$ for all $\lambda$. Hence $Z \subseteq \mathcal{D}$.

Remark. Contrary to the notion of Bayesian rationality, rationality* relies on the simpler and more elementary decision rule of "payoff undominance." 13 One remarkable feature of rationality* is that it does not require a probabilistic apparatus. This approach can be also supported by Mariotti et al.'s (2005) work on the construction of Harsanyi's type where a player has a "belief" represented by a non-probabilistic set of states. To characterize the point-rationalizability concept, Mariotti (2003) offered a similar type notion of "rationality" without using a probabilistic apparatus - i.e., $i$ is pointrational at $\omega$ iff $\exists \omega^{\prime} \in P_{i}(\omega)$ such that $u_{i}\left(\chi_{i}(\omega), \chi_{-i}\left(\omega^{\prime}\right)\right) \geqslant u_{i}\left(x_{i}, \chi_{-i}\left(\omega^{\prime}\right)\right)$ for all $x_{i} \in X_{i}$.

\section{$4 \quad$ IESDS $^{*}$ and Nash Equilibrium}

As Nash (1950, p. 292) pointed out, "no equilibrium point can involve a dominated strategy". Nash equilibrium is clearly related to the notion of dominance. In this section we study the relationship between Nash equilibrium and IESDS*.

We have shown in Corollary 1 that every Nash equilibrium survives IESDS* and hence remains a Nash equilibrium in the reduced game after the iterated elimination procedure. However, a Nash equilibrium in the reduced game after the iterated elimination procedure may fail to be a Nash equilibrium in the original game. DS showed by examples (see their Exam-

\footnotetext{
${ }^{13}$ From a decision-theoretic point of view, although subjective expected utility is undoubtedly the dominant model in economics, many economists would probably view axioms such as "transitivity" or "monotonicity" as more basic tenets of rationality than the Sure-Thing-Principle and other components of the Savage (1954) model. The notion of rationality proposed here is based solely upon such a more basic axiom of "monotonicity" on preferences. See Luce and Raiffa (1957, Chapter 13) and Epstein (1997) for more discussions.
} 
ples 1, 4, 5, and 8) that their IESDS procedure can generate spurious Nash equilibria. Since our IESDS* has more elimination power, it can be easily verified that if we apply our IESDS* to DS's examples, there are no spurious Nash equilibria. Despite this happy outcome, the following example shows that IESDS* can generate spurious Nash equilibria.

Example 4. Consider a two-person symmetric game: $\mathcal{G} \equiv\left(N,\left\{X_{i}\right\}_{i \in N},\left\{u_{i}\right\}_{i \in N}\right)$, where $N=\{1,2\}, X_{1}=X_{2}=[0,1]$, and for all $x_{i}, x_{j} \in[0,1], i, j=1,2$, and $i \neq j$ (cf. Fig. 2)

$$
u_{i}\left(x_{i}, x_{j}\right)= \begin{cases}1, & \text { if } x_{i} \in[1 / 2,1] \text { and } x_{j} \in[1 / 2,1] \\ 1+x_{i}, & \text { if } x_{i} \in[0,1 / 2) \text { and } x_{j} \in(2 / 3,5 / 6) . \\ x_{i}, & \text { otherwise }\end{cases}
$$

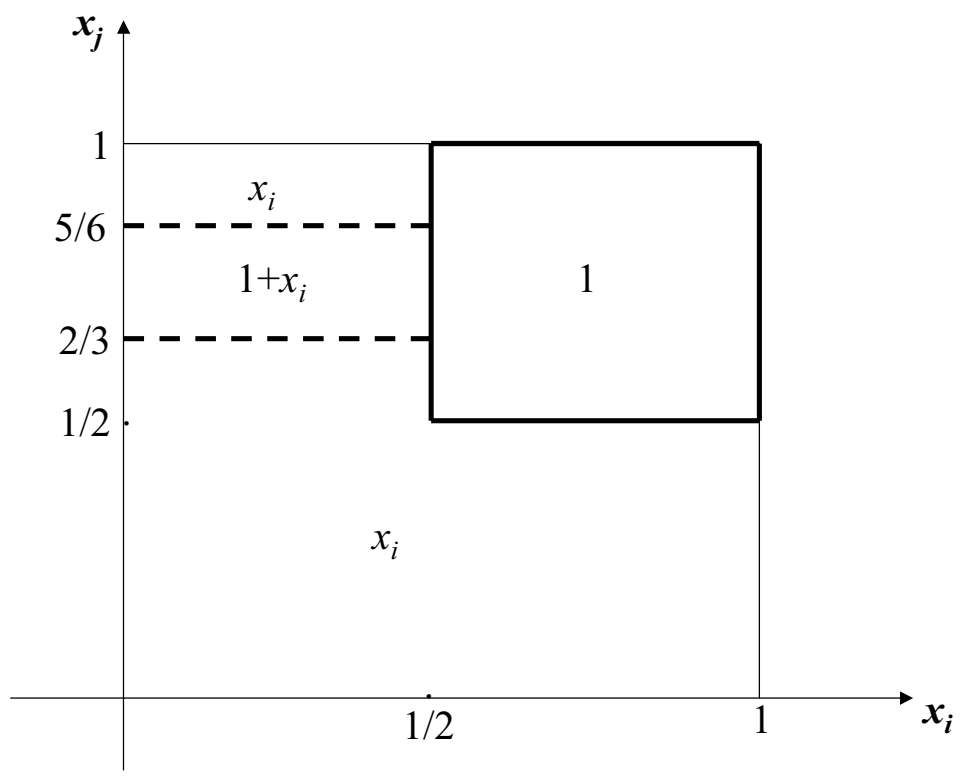

Fig. 2. Payoff function $u_{i}\left(x_{i}, x_{j}\right)$.

It is easily verified that $\mathcal{D}=[1 / 2,1] \times[1 / 2,1]$ since any $y_{i} \in[0,1 / 2)$ is dominated. That is, IESDS* leaves the reduced game $\left.\mathcal{G}\right|_{\mathcal{D}} \equiv\left(N,\left\{\mathcal{D}_{i}\right\}_{i \in N},\left\{\left.u_{i}\right|_{\mathcal{D}}\right\}_{i \in N}\right)$ 
that cannot be further reduced, where $\left.u_{i}\right|_{\mathcal{D}}$ is the payoff function $u_{i}$ restricted on $\mathcal{D}$. Clearly, $\mathcal{D}$ is the set of Nash equilibria in the reduced game $\left.\mathcal{G}\right|_{\mathcal{D}}$ since $\left.u_{i}\right|_{\mathcal{D}}$ is a constant function. However, it is easy to see that the set of Nash equilibria in game $\mathcal{G}$ is $\left\{x \in \mathcal{D} \mid x_{1}, x_{2} \notin(2 / 3,5 / 6)\right\}$. Thus, IESDS* generates spurious Nash equilibria $x \in \mathcal{D}$ where some $x_{i} \in(2 / 3,5 / 6)$.

Remark. Example 4 belongs to Reny's (1999) class of games for which a Nash equilibrium exists (in this class of games, the player set is finite, the strategy sets are compact and convex, payoff functions are quasi-concave in own strategies, and a condition called "better-reply security" holds). To see that game $\mathcal{G}$ in Example 4 belongs to Reny's class of games, let us check the better-reply secure property. Recall that better-reply security means that "for every non equilibrium strategy $x^{*}$ and every payoff vector limit $u^{*}$ resulting from strategies approaching $x^{*}$, some player $i$ has a strategy yielding a payoff strictly above $u_{i}^{*}$ even if the others deviate slightly from $x^{*}$ (Reny 1999, p. 1030)". Let $\epsilon>0$ be sufficiently small. We consider the following two cases:

(1) If $x^{*} \notin \mathcal{D}$, then some $x_{i}^{*}<1 / 2$. Thus, $i$ can secure payoff $x_{i}^{*}+\epsilon>u_{i}^{*}=$ $x_{i}^{*}\left(\right.$ if $\left.x_{j}^{*} \notin(2 / 3,5 / 6)\right)$ or $x_{i}^{*}+1+\epsilon>u_{i}^{*}=x_{i}^{*}+1$ (if $x_{j}^{*} \in(2 / 3,5 / 6)$ ) by choosing a strategy $x_{i}^{*}+\epsilon$.

(2) If $x^{*} \in \mathcal{D}$, then some $x_{i}^{*} \in(2 / 3,5 / 6)$ and $x_{j}^{*} \geq 1 / 2$. We distinguish two subcases: $(2.1) x_{j}^{*}>1 / 2$. As $x_{i}^{*}$ lies in an open interval $(2 / 3,5 / 6)$, $j$ can secure payoff $1+x_{j}>1$ by choosing a strategy $x_{j} \in(0,1 / 2)$. $(2.2) x_{j}^{*}=1 / 2$. In this subcase, the limiting vector $u^{*}$ depends on how $x$ approaches $x^{*}$. We must distinguish two subsubcases. (2.2.1) $u^{*}=(1,1)$. Similarly to $(2.1), j$ can secure payoff $1+x_{j}>1$ by choosing a strategy $x_{j} \in(0,1 / 2)$. (2.2.2) The limiting payoff vector is $u^{*}=\left(x_{i}^{*}, 3 / 2\right)$ even though the actual payoff vector at $x^{*} \in \mathcal{D}$ is $(1,1)$. Thus, $i$ can secure payoff $x_{i}^{*}+\epsilon>u_{i}^{*}=x_{i}^{*}$ by choosing a strategy $x_{i}^{*}+\epsilon$, 
since for any $x_{j}$ that deviates slightly from $1 / 2$,

$$
u_{i}\left(x_{i}^{*}+\epsilon, x_{j}\right)=\left\{\begin{array}{ll}
x_{i}^{*}+\epsilon, & \text { if } x_{j}<1 / 2 \\
1, & \text { if } x_{j} \geq 1 / 2
\end{array} .\right.
$$

Moreover, the player set is finite, strategy set $X_{i}=[0,1]$ is compact and convex, and payoff function $u_{i}\left(\cdot, x_{j}\right)$ is quasi-concave and bounded. This example shows that IESDS* can generate spurious Nash equilibria in the class of Reny's better-reply secure games. Observe that in Example $4, u_{i}\left(., x_{j}\right)$ has no maximizer for $x_{j} \in(2 / 3,5 / 6)$.

We next provide a sufficient and necessary condition under which IESDS* preserves Nash equilibria. Consider a game $\mathcal{G} \equiv\left(N,\left\{X_{i}\right\}_{i \in N},\left\{u_{i}\right\}_{i \in N}\right)$. For a given subset $Y \subseteq X$, we say that $\mathcal{G}$ has "well-defined best replies on $Y$ " if for every $i \in N$ and for every $y_{-i} \in Y_{-i}$, there is $x_{i} \in X_{i}$ that maximizes $u_{i}\left(., y_{-i}\right)$. We say that $\mathcal{G}$ is "dominance-solvable" if IESDS* leads to a unique strategy choice for each player. ${ }^{14}$ The following Theorem 5 states that IESDS* generates no spurious Nash equilibria if, and only if, best replies are well defined on the set of Nash equilibria in the reduced game after performing the IESDS* procedure. Moreover, in one-person games and dominancesolvable games, Nash equilibria can be solved by our IESDS* procedure. Formally, let $\mathcal{N} \mathcal{E}$ denote the set of Nash equilibria in $\mathcal{G}$, and let $\left.\mathcal{N} \mathcal{E}\right|_{\mathcal{D}}$ denote the set of Nash equilibria in the reduced game $\left.\mathcal{G}\right|_{\mathcal{D}} \equiv\left(N,\left\{\mathcal{D}_{i}\right\}_{i \in N},\left\{\left.u_{i}\right|_{\mathcal{D}}\right\}_{i \in N}\right)$, where $\left.u_{i}\right|_{\mathcal{D}}$ is the payoff function $u_{i}$ restricted on $\mathcal{D}$.

Theorem $5 \mathcal{N E}=\left.\mathcal{N} \mathcal{E}\right|_{\mathcal{D}}$ iff $\mathcal{G}$ has well-defined best replies on $\left.\mathcal{N} \mathcal{E}\right|_{\mathcal{D}}$. Moreover, $\mathcal{N} \mathcal{E}=\mathcal{D}$ if $\mathcal{G}$ is a one-person or dominance-solvable game.

Proof. By Corollary 1, it suffices to show $\left.\mathcal{N E}\right|_{\mathcal{D}} \subseteq \mathcal{N E}$ if, and only if, $\mathcal{G}$ has well-defined best replies on $\left.\mathcal{N} \mathcal{E}\right|_{\mathcal{D}}$. Suppose $\mathcal{G}$ has well-defined best replies

\footnotetext{
${ }^{14}$ For example, the standard Cournot game (Moulin, 1984), Bertrand oligopoly with differentiated products, the arms-race games (Milgrom and Roberts, 1990), and global games (Carlsson and van Damme, 1993).
} 
on $\left.\mathcal{N} \mathcal{E}\right|_{\mathcal{D}}$. Let $\left.x^{*} \in \mathcal{N} \mathcal{E}\right|_{\mathcal{D}}$. Then, for every $i \in N, u_{i}\left(x_{i}^{* *}, x_{-i}^{*}\right) \geq u_{i}\left(x_{i}, x_{-i}^{*}\right)$ for some $x_{i}^{* *} \in X_{i}$ and all $x_{i} \in X_{i}$ and, hence, $x_{i}^{* *}$ is not dominated given $\mathcal{D}$. Since $x^{*} \in \mathcal{D}$, by Theorem $3,\left(x_{i}^{* *}, x_{-i}^{*}\right) \in \mathcal{D}$. Since $\left.x^{*} \in \mathcal{N} \mathcal{E}\right|_{\mathcal{D}}$, it follows that $u_{i}\left(x_{i}^{*}, x_{-i}^{*}\right) \geq u_{i}\left(x_{i}^{* *}, x_{-i}^{*}\right) \geq u_{i}\left(x_{i}, x_{-i}^{*}\right)$ for all $x_{i} \in X_{i}$. That is, $x^{*} \in \mathcal{N} \mathcal{E}$. Thus, $\left.\mathcal{N} \mathcal{E}\right|_{\mathcal{D}} \subseteq \mathcal{N} \mathcal{E}$. Conversely, suppose $\left.\mathcal{N} \mathcal{E}\right|_{\mathcal{D}} \subseteq \mathcal{N} \mathcal{E}$. Therefore, for every $i \in N$ and for every $\left.x^{*} \in \mathcal{N} \mathcal{E}\right|_{\mathcal{D}}, u_{i}\left(x_{i}^{*}, x_{-i}^{*}\right) \geq u_{i}\left(x_{i}, x_{-i}^{*}\right)$ for all $x_{i} \in X_{i}$. That is, $\mathcal{G}$ has well-defined best replies on $\left.\mathcal{N} \mathcal{E}\right|_{\mathcal{D}}$.

By Theorem $3, \mathcal{D}$ is a stable set. Therefore, for every $i \in N$ and for every $x^{*} \in \mathcal{D}, u_{i}\left(x^{*}\right) \geq u_{i}\left(x_{i}, x_{-i}^{*}\right)$ for all $x_{i} \in X_{i}$ if $\mathcal{G}$ is a one-person or dominance-solvable game. Thus, every $x^{*} \in \mathcal{D}$ is a Nash equilibrium. By Corollary $1, \mathcal{N E}=\mathcal{D}$.

Remark. The first part of Theorem 5 offers a sufficient and necessary condition of a spurious Nash equilibrium, which asserts that a spurious Nash equilibrium appears if, and only if, the best reply for some player at that spurious Nash equilibrium is not well defined. It is easy to see the first part result of Theorem 5 is true also for DS's IESDS procedure - i.e., DS's (2002) Theorem 2 can be improved in the same manner. It is worthy of note that the weak condition of "well-defined best replies" is only for the set of Nash equilibria in the reduced game. We would also like to emphasize that the second part of Theorem 5 is not true for DS's IESDS procedure. To see this, consider again the one-person game in the Introduction. Clearly, no Nash equilibrium exists in the example. Because a single strategy $x \in(0,1)$ can survive DS's IESDS procedure, $\mathcal{H}=\{x\} \neq \mathcal{N} \mathcal{E}$. This demonstrates that DS's IESDS procedure can generate spurious Nash equilibria in the simplest class of one-person games. ${ }^{15}$

Many important economic applications such as the Cournot game are

\footnotetext{
${ }^{15}$ DS's (2002) Example 1 shows that their IESDS procedure can generate spurious Nash equilibria in a "dominance-solvable" game. In particular, that example satisfies the weak condition of "well-defined best replies" in Theorem 5 .
} 
dominance-solvable. As DS's Examples 3 and 8 illustrate, their IESDS procedure may fail to yield a maximal reduction and may produce spurious Nash equilibria in the Cournot game. By our Theorem 5, Nash equilibrium can be solved by our IESDS* in the class of dominance-solvable games. The following example, taken from DS's Example 3, illustrates this point.

Example 5 (Cournot competition with outside wager). Consider a threeperson game $\mathcal{G} \equiv\left(N,\left\{X_{i}\right\}_{i \in N},\left\{u_{i}\right\}_{i \in N}\right)$, where $N=\{1,2,3\}, X_{1}=X_{2}=$ $[0,1], X_{3}=\{\alpha, \beta\}$, and for all $x_{1}, x_{2}$, and $x_{3}, u_{1}\left(x_{1}, x_{2}, x_{3}\right)=x_{1}\left(1-x_{1}-x_{2}\right)$, $u_{2}\left(x_{1}, x_{2}, x_{3}\right)=x_{2}\left(1-x_{1}-x_{2}\right)$, and

$$
\left\{\begin{array}{ll}
u_{3}\left(x_{1}, x_{2}, \alpha\right)>u_{3}\left(x_{1}, x_{2}, \beta\right), & \text { if }\left(x_{1}, x_{2}\right)=(1 / 3,1 / 3) \\
u_{3}\left(x_{1}, x_{2}, \alpha\right)<u_{3}\left(x_{1}, x_{2}, \beta\right), & \text { otherwise }
\end{array} .\right.
$$

This game is dominance-solvable since our IESDS* yields $(1 / 3,1 / 3, \alpha)$, which is the unique Nash equilibrium. By contrast, DS's IESDS procedure fails to give a maximal reduction since, within the first infinite ordinal (natural numbers), no sequence of elimination can eliminate the strategy $\beta$ for player 3.

We close this section by listing the "preserving Nash equilibria" results for our IESDS* in some classes of games commonly discussed in the literature. These results follow immediately from Theorem 5 .

Corollary 4. $\mathcal{D}$ preserves the (nonempty) set of Nash equilibria in the following classes of games $\mathcal{G} \equiv\left(N,\left\{X_{i}\right\}_{i \in N},\left\{u_{i}\right\}_{i \in N}\right)$ :

(i) (Debreu 1952; Fan 1966; Glicksberg 1952). $X_{i}$ is a nonempty, convex, and compact Hausdorff topological vector space; $u_{i}$ is quasiconcave on $X_{i}$ and continuous on $X_{i} \times X_{-i}$.

(ii) (Dasgupta and Maskin 1986). $N$ is a finite set; $X_{i}$ is a nonempty, convex, and compact space in a finite-dimensional Euclidian space; $u_{i}$ 
is quasi-concave on $X_{i}$, uppersemicontinuous on $X_{i} \times X_{-i}$, and graph continuous.

(iii) (Topkis 1979; Vives 1990; Milgrom and Roberts 1990). $\mathcal{G}$ is a supermodular game such that $X_{i}$ is a complete lattice; and $u_{i}$ is order upper-semi-continuous on $X_{i}$ and is bounded above.

Proof. By the Generalized Weierstrass Theorem (see, e.g., Aliprantis and Border 1999, 2.40 Theorem), the best replies are well-defined for the compact and own-uppersemicontinuous games. By Milgrom and Roberts's (1990) Theorem 1, the best replies are well-defined for the supermodular games in which strategy spaces are complete lattices. By Theorem 5, IESDS* preserves the (nonempty) set of Nash equilibria for these classes of games in Corollary 4 .

\section{Concluding Remarks}

We have presented a new notion of IESDS* that can be used in general games. As Milgrom and Roberts (1990, p. 1269) showed, the concept of IESDS* can be supported by an appealing adaptive learning process. We have shown that IESDS* is always a well-defined order independent procedure, and that it can be used to identify Nash equilibrium in dominance-solvable games; e.g., the Cournot competition, Bertrand oligopoly with differentiated products, and the arms-race games. In addition, we have characterized IESDS* as the largest stable set, which suggests itself an interesting alternative definition of IESDS*, and we have also provided an epistemic characterization for IESDS* by the notion of rationality* in terms of payoff-dominance.

Our IESDS* procedure avoids many of the problems that arise when using DS's IESDS procedure in general games, which are noted by DS (2002). Some comparisons between IESDS* and DS's IESDS are summarized in Table 1. 


\begin{tabular}{|c|c|c|}
\hline & $\mathcal{C C}$ games & $\neg \mathcal{C C}$ games \\
\hline \multirow{5}{*}{ IESDS } & \multirow{5}{*}{$\begin{array}{l}\cdot \text { well-defined } \\
\text { - nonempty \& order-independent } \\
\text {. no spurious Nash }\end{array}$} & $\cdot \neg$ well-defined \\
\hline & & . $\neg$ nonempty or $\neg$ order-independent (if well defined) \\
\hline & & · spurious Nash e.g. in Reny's better-reply secure games, \\
\hline & & and even in 1-person/dominance-solvable games \\
\hline & & . no spurious Nash if best replies are well defined \\
\hline \multirow{5}{*}{ IESDS* } & \multirow{5}{*}{$\cdot \mathrm{IESDS}^{*}=\mathrm{IESDS}$} & $\cdot$ well-defined \\
\hline & & · nonempty (if Nash exists) \& order-independent \\
\hline & & - spurious Nash e.g. in Reny's better-reply secure games, \\
\hline & & but not in 1-person/dominance-solvable games \\
\hline & & - no spurious Nash iff best replies are well defined on $\left.\mathcal{N} \mathcal{E}\right|_{\mathcal{D}}$ \\
\hline
\end{tabular}

Table 1: DS's IESDS vs. IESDS* $(\mathcal{C C}=$ Compact \& Continuous; $\neg=$ logical negation $)$

Many game theorists do not recommend iterated elimination of weakly dominated strategies (IEWDS) as a solution concept, and one important reason is that order matters for that procedure in some games (see, e.g., Marx and Swinkels 1997). This criticism can also be applied to DS's IESDS procedure, but not to our IESDS* procedure. More importantly perhaps, IEWDS is troublesome in being interpreted as an implication of common knowledge of "cautious" rationality; see, e.g., Borgers and Samuelson (1992), Brandenburger et al.'s (2004), and Samuelson (1992, 2004). In contrast, we can establish a sufficient and necessary epistemic condition for IESDS*. Our IESDS* procedure can be interpreted as an implication of common knowledge of rationality* that is based upon a more elementary behavioral assumption.

We would like to point out that the problem of "spurious Nash equilibria" for our IESDS* procedure appears to be less severe than that for DS's IESDS procedure. In all the examples provided by DS (2002) to illustrate that their procedure creates spurious Nash equilibria, the use of our IESDS* procedure does not generate any spurious Nash equilibria. Indeed, we have shown that in our IESDS* procedure, this problem never arises both in oneperson games and in dominance-solvable games. We have demonstrated by Example 4 that IESDS* can generate spurious Nash equilibria in general 
games. One major feature of Example 4 is that there is no best reply at each of spurious Nash equilibria in the reduced game (and thus IESDS* creates spurious Nash equilibria). The creation of spurious Nash equilibria by IESDS seems to be a generic property of games that are not compact and own-uppersemicontinuous.

Finally, we would like to mention that in a related paper, Apt (2005) investigated the problem of order independence for "(possibly transfinite) iterated elimination of never-best replies." Apt (2005, Theorem 4.2) also showed a similar type of order-independent result and demonstrated that the result fails to hold for some different iteration procedures.

\section{Acknowledgments}

This paper partially done while the third author was visiting the Institute for Mathematical Sciences, National University of Singapore in 2005. We are grateful to an associate editor and two anonymous referees for their insight

and useful comments and suggestions. We would like to thank Krzysztof R. Apt, Y. Stephen Chiu, Youngsub Chun, Kim-Sau Chung, Yossi Greenberg, Tai-Wei Hu, Chenying Huang, Wei-Torng Juang, Huiwen Koo, FanChin Kung, In Ho Lee, Kim Long, Stephen Morris, Man-Chung Ng, Yeneng Sun, Siyang Xiong, Licun Xue, and Chun-Hsien Yeh for helpful discussions and comments. We especially thank Martin Dufwenberg for his encouragement and helpful comments. We thank participants in seminars at Academia Sinica, McGill University, National University of Singapore, Seoul National University, University of Hong Kong, the 16th International Conference on Game Theory, the 2005 Midwest Economic Theory conference, and the 2nd Pan-Pacific Conference on Game Theory. Financial supports from the National Science Council of Taiwan, and from SSHRC of Canada are gratefully acknowledged. The usual disclaimer applies. 


\section{REFERENCES}

Aliprantis, C., Border, K., 1999. Infinite Dimensional Analysis. Springer-Verlag, Berlin.

Apt, K.R., 2005. Order independence and rationalizability. Mimeo, National University of Singapore.

Aumann, R., 1976. Agreeing to disagree. Ann. Stat. 4, 1236-1239.

Aumann, R., 1987. Correlated equilibrium as an expression of Bayesian rationality. Econometrica 55, 1-18.

Aumann, R., 1995. Backward induction and common knowledge of rationality. Games Econ. Behav. 8, 6-19.

Aumann, R., 1999. Interactive epistemology I: Knowledge. Int. J. Game Theory $28,263-300$.

Aumann, R., Brandenburger, A., 1995. Epistemic conditions for Nash equilibrium. Econometrica 63, 1161-1180.

Bergemann, D., Morris, S., 2005. Robust implementation: The role of large type space. Cowles Foundation Discussion Paper No. 1519.

Bernheim, B.D., 1984. Rationalizable strategic behavior. Econometrica 52, 10071028.

Borgers, T., 1993. Pure strategy dominance. Econometrica 61, 423-430.

Borgers, T., Samuelson, L., 1992. "Cautious" utility maximization and iterated weak dominance. Int. J. Game Theory 21, 13-25.

Brandenburger, A., Dekel, E., 1987. Rationalizability and correlated equilibrium. Econometrica 55, 1391-1402.

Brandenburger, A., Friedenberg, A., Keisler, H.J., 2004. Admissibility in games. Mimeo, New York University.

Carlsson, H., van Damme, E., 1993. Global games and equilibrium selection. Econometrica 61, 989-1018.

Chen, Y.C., Luo, X., 2003. A unified approach to information, knowledge, and stability. Mimeo, Academia Sinica.

Dasgupta, P., Maskin, E., 1986. The existence of equilibrium in discontinuous economic games, I: Theory. Rev. Econ. Stud. 53, 1-26. 
Debreu, G., 1952. A social equilibrium existence theorem. Proc. Nat. Acad. Sci. U.S.A. $38,386-393$.

Dufwenberg, M., Stegeman, M., 2002. Existence and uniqueness of maximal reductions under iterated strict dominance. Econometrica 70, 2007-2023.

Epstein, L., 1997. Preference, rationalizability and equilibrium. J. Econ. Theory $73,1-29$.

Fan, K., 1966. Application of a theorem concerning sets with convex sections. Math. Ann. 163, 189-203.

Gilboa, I., Kalai, E., Zemel, E., 1990. On the order of eliminating dominated strategies. Oper. Res. Letters 9, 85-89.

Glicksberg, I.L., 1952. A further generalization of the Kakutani fixed point theorem with application to Nash equilibrium points. Proc. Amer. Math. Soc. $38,170-174$.

Jackson, M.O., 1992. Implementation of undominated strategies: A look at bounded mechanism. Rev. Econ. Stud. 59, 757-775.

Lipman, B.L., 1994. A note on the implication of common knowledge of rationality. Games Econ. Behav. 6, 114-129.

Luce, R.D., Raiffa, H., 1957. Games and Decisions: Introduction and Critical Survey. John Wiley \& Sons, NY.

Luo, X., 2001. General systems and $\varphi$-stable sets - a formal analysis of socioeconomic environments. J. Math. Econ. 36, 95-109.

Mariotti, T., 2003. Hierarchies of compact beliefs and rationalizable behavior. Econ. Letters 79, 199-204.

Mariotti, T., Meier, M., Piccione, M., 2005. Hierarchies of beliefs for compact possibility models. J. Math. Econ. 41, 303-324.

Marx, L.M., Swinkels, J.M., 1997. Order dependence of iterated weak dominance. Games Econ. Behav. 18, 219-245.

Milgrom, P., Roberts, J., 1990. Rationalizability, learning, and equilibrium in games with strategic complementarities. Econometrica 58, 1255-1278.

Milgrom, P., Roberts, J., 1996. Coalition-proofness and correlation with arbitrary communication possibilities. Games Econ. Behav. 17, 113-128. 
Morris, S., Shin, H.S., 2003. Global games: theory and applications. In: Dewatripont, M., Hansen, L., Turnoksky, S. (Eds.). Advances in Economic Theory and Econometrics: Proceedings of the Eighth World Congress of the Econometric Society. Cambridge University Press, pp. 56-114.

Moulin, H., 1984. Dominance solvability and Cournot stability. Math. Soc. Sci. 7, 83-102.

Nash, J., 1951. Non-cooperative games. Ann. Math. 54, 286-295.

Osborne, M.J., Rubinstein, A., 1994. A Course in Game Theory. The MIT Press, MA.

Pearce, D.G., 1984. Rationalizable strategic behavior and the problem of perfection. Econometrica 52, 1029-1051.

Reny, P., 1999. On the existence of pure and mixed strategy Nash equilibrium in discontinuous games. Econometrica 67, 1029-1056.

Ritzberger, K., 2002. Foundations of Non-Cooperative Game Theory. Oxford University Press, Oxford.

Stegeman, M., 1990. Deleting strictly dominated strategies, Working Paper 1990/16, University of North Carolina.

Samuelson, L., 1992. Dominated strategies and common knowledge. Games Econ. Behav. 4, 284-313.

Samuelson, L., 2004. Modeling knowledge in economic analysis. J. Econ. Lit. $42,367-403$.

Savage, P., 1954. The Foundations of Statistics. Wiley, NY.

Suppes, P., 1972. Axiomatic Set Theory. Dover, NY.

Tan, T., Werlang, S., 1988. The Bayesian foundations of solution concepts of games. J. Econ. Theory 45, 370-391.

Topkis, D., 1979. Equilibrium points in nonzero-sum $n$-person submodular games. SIAM J. Control Optim. 17, 773-787.

Vives, X., 1990. Nash equilibrium with strategic complementarities. J. Math. Econ. 19, 305-321. 